Primljen / Received: 10.6.2013. Ispravljen / Corrected: 24.7.2013.

Prihvaćen / Accepted: 1.8.2013. Dostupno online / Available online: 10.9.2013.

\section{Generalized minimal nets in form finding of prestressed cable nets}

\section{Authors:}

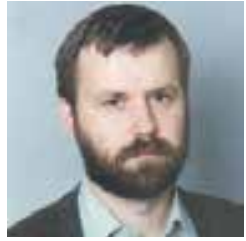

Prof. Krešimir Fresl, PhD. CE

University of Zagreb

Faculty of Civil Engineering

fresl@grad.hr

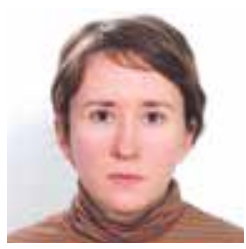

Petra Gidak, B.Sc. CE

University of Zagreb

Faculty of Civil Engineering

pgidak@grad.hr

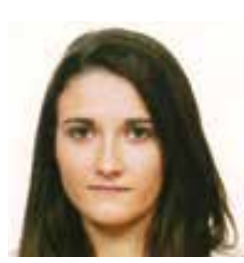

Romana Vrančić, student

University of Zagreb

Faculty of Civil Engineering

rvrancic@student.grad.hr
Original scientific paper

\section{Krešimir Fresl, Petra Gidak, Romana Vrančić}

\section{Generalized minimal nets in form finding of prestressed cable nets}

Form finding problem of prestressed cable structures is formulated as a variational problem whose solutions are minimal and generalized minimal nets. Kinematic constrains are introduced that allow assignment of chosen lengths to elements. Application of NewtonKrylov methods for solving nonlinear equations of equilibrium is analysed. Iterative application of force density method, where force densities in each iteration step are determined based on given conditions and results of previous step, is described and analyzed.

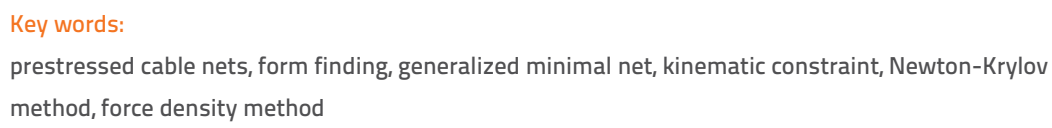

Krešimir Fresl, Petra Gidak, Romana Vrančić

Izvorni znanstveni rad

\section{Poopćene minimalne mreže u oblikovanju prednapetih konstrukcija od užadi}

Problem nalaženja oblika prednapetih konstrukcija od užadi formuliran je kao varijacijski problem čija su rješenja minimalne i poopćene minimalne mreže. Uvedena su kinematička ograničenja koja omogućuju zadavanje duljina odabranih štapova. Analizirana je primjena Newton-Krilovljevih metoda u rješavanju nelinearnih jednadžbi ravnoteže te je opisana i analizirana iteracijska primjena metode gustoća sila u kojoj se gustoće sila u nekom koraku određuju na temelju zadanih uvjeta i rezultata prethodnoga koraka.

Ključne riječi:

prednapeta konstrukcija od užadi, nalaženje oblika, poopćena minimalna mreža, kinematičko ograničenje, Newton-Krylovljeva metoda, metoda gustoća sila

Wissenschaftlicher Originalbeitrag

Krešimir Fresl, Petra Gidak, Romana Vrančić

\section{Verallgemeinerte Minimalnetze im Entwurf vorgespannter Seilkonstruktionen}

Das Problem der Formfindung vorgespannter Seilkonstruktionen ist als Variationsproblem formuliert, dessen Lösungen Minimalnetze und verallgemeinerte Minimalnetze darstellen. Kinematische Einschränkungen, die das Bestimmen der Länge ausgewählter Stabelemente ermöglichen, sind eingeführt. Der Einsatz von Newton-Krylov-Verfahren beim Lösen nichtlinearer Gleichgewichtsgleichungen ist analysiert und die iterative Anwendung der Kraftdichtemethode, bei der die Kraftdichte in jedem Iterationsschritt aufgrund der gegebenen Konditionen und der Resultate im vorherigen Schritt ermittelt wird, ist ebenfalls beschrieben und untersucht.

\section{Schlüsselwörter:}

vorgespannte Seilkonstruktion, Formfindung, verallgemeinertes Minimalnetz, kinematische

Einschränkung, Newton-Krylov-Verfahren, Kraftdichtemethode 


\section{Introduction}

Most structural elements take over and transfer loads through various stresses and their combinations: tension, compression and shear. This results in complex distribution of stresses that can be seen in photoelastic experiments. However, the cable has a negligible flexural stiffness, insufficient to enable transfer of greater forces applied perpendicular to its axis. In addition, it can not take over compressive forces. That is why, when subjected to load, the cable changes its shape to enable development of balanced tensile forces, with uniform distribution of tensile stresses along the cross-sectional area. That is why cables significantly change the equilibrium configuration if forces acting on them change their position, direction, or orientation. On the other hand, as all cross-sectional points are equally stressed, cables are highly efficient in transferring forces.

As the cable, taken by itself, changes the equilibrium configuration at the change of load, the shape of flexible cable structures is maintained by arranging cables into a net that forms an anticlastic surface (surface with negative Gaussian curvature). In simplest cases, there are two families of cables: concave cables assume load (dominant gravitational load) while convex cables, spread approximately perpendicular to concave cables, are used for stabilisation. In addition, cables must be prestressed as the tensile stress in the entire net must be maintained at all load combinations. For instance, cable families may exchange their roles in case of wind action. Consequently, the geometric shape and prestressed force values are of crucial significance for the static (and, of course, dynamic) behaviour of flexible cable structures - the anticlastic shape and prestressing provide the so called geometric stiffness to such structures. Conversely, their shape is determined by the laws of statics: the geometric shape is a direct reflection of the force system in equilibrium; an arbitrarily selected shape and prestressing force values will hardly meet equilibrium requirements. Here we are in presence of a certain paradox: seemingly free, scattered, almost organic forms of prestressed cable structures (Figure 1) are in fact determined with stringent, inexorable and unyielding laws of statics [1].

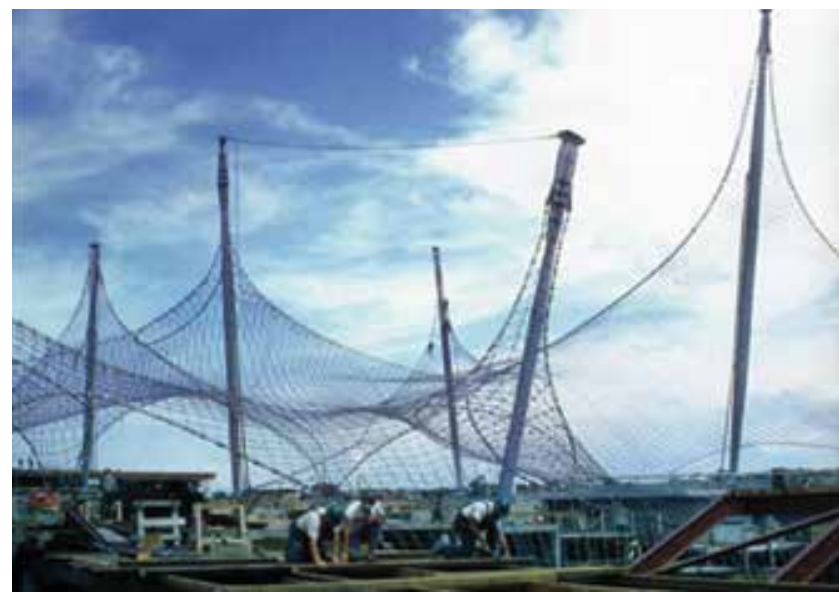

Figure 1. Frei Otto: German pavilion at the Expo '67 in Montreal
The first phase in the design of a prestressed cable structure is therefore the definition of its form, i.e. form finding, before application of the service load, most often with the neglect of the structure's own weight. The term form finding implies determination of an initial equilibrium configuration that comprises the geometric shape of the structure and prestressing force values.

Until the end of the 1960s, the only way to define form was through establishment of physical models. Frei Otto used models made of fabric and wire and soapsuds membranes for his projects [1]. Although physical models give a useful insight into the behaviour of flexible structures, it is very difficult, even with photogrammetric procedures, to determine with sufficient accuracy coordinates of individual points, not to mention a more accurate determination of force and stress values. That is why computational models and methods have started to develop quite early.

A detailed presentation of different form finding procedures for prestressed structures is given in [2,3], and so we will give only a short overview, by selectively mentioning only some of these procedures.

One of first computational methods is an extension of the geometrically nonlinear calculation of hinged bar elements by means of the displacement method: the trial configuration deflects when subjected to nonuniform prestressing forces (great displacements with small deformations) and thus it gradually approaches an equilibrium configuration [4]. Unfortunately, displacements and forces are hard to control during this process, and so compressive forces may occur at some parts of the cable. In addition, as this is a displacement method variant, the constitutive relation between the displacement and force should be assumed, but this relation does not have to correspond to the real material because, as will be demonstrated, the form finding is a static problem.

In the force density method, which was developed in the early 1970 s by H.-J. Schek, K. Linkwitz and their associates, the nonlinear problem of form finding was linearized through a "different association" of values which has lead to the notion of force density [5-7]. The paper [7] contains derivation of the

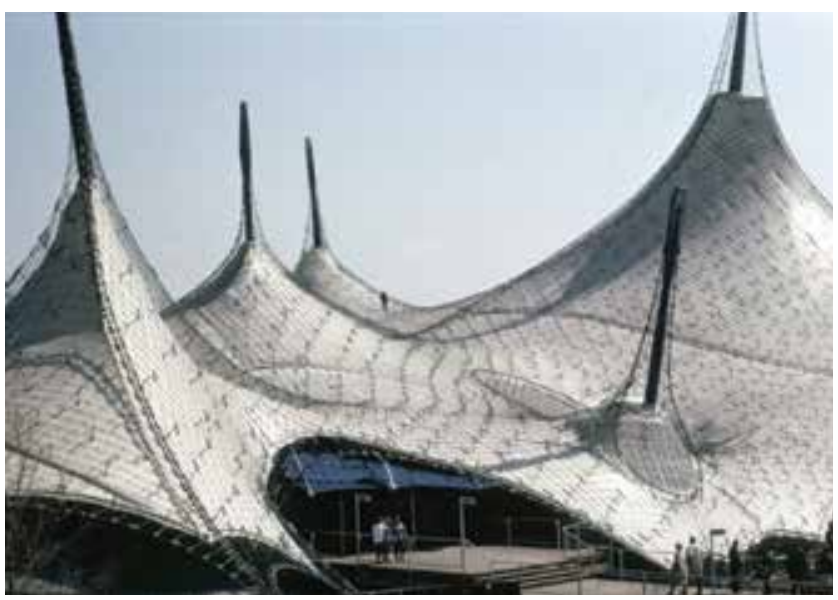


method's equations based on the full system of equilibrium equations, compatibility equations, and constitutive equations, with the use of the least-squares method. The force density methods will be described in more detail in Section 7, but the equations will be derived directly, from equilibrium equations. This method is often applied even today, and its numerous extensions and generalisations have so far been developed. The nonlinear variant of the force density method, allowing for definition of additional constraints, has been introduced already in [5].

The form finding problem can also be understood as a variational problem, i.e. a minimum finding problem [8-10] or, as will be shown in Section 5, as a more general problem of finding stationary points of some functions. We consider that this is the most natural approach.

In paper [11] the authors assert that "basic parameters" for finding the form of fabric structures are: surface topology, surface geometry, geometric boundary conditions, stress distribution, and the volume and area forces. Although included in basic parameters, the volume and area forces (self-weight, load) are seldom (as already indicated) introduced in calculation in the form finding phase. The form finding tasks are formulated depending on which parameters are defined, and which ones are considered to be the unknowns. In the context of cable structures, we will speak of the net topology and net geometry and, instead of stress distribution, we will speak of values of prestressing forces in cables.

\section{Computational model of cable nets}

For the purposes of forming the computational model of cable nets, it can be assumed that cables are fully flexible and that they are weightless. As only the form finding phase will be considered, no external load will be applied. If prestressing tensile forces are introduced into cables, contact forces will act in between them at cable crossing points. If cables can slide one over another without friction, these forces will be normal to contact surfaces, while in case of friction tangential components will also be introduced. As contact forces act on negligibly small areas, the contact forces shall be taken to be concentrated. Because of the assumed absolute flexibility, the cable subjected to concentrated forces only shall assume the form of planar or spatial polygonal line, in such a way that sections between points in which cable cross will be straight (Figure 2). Although due to cable thickness their axis do not intersect at crossing points, it will be assumed that these crossing points are geometrical points, i.e. that cable axes actually intersect at such points. Taking into account these assumptions, it can be supposed in the computational model that cable sections between the crossing points are hinged bar elements, and that the crossing points are hinged nodes to which elements are centrically attached. The hinged bar system can also be used as computational model in the phase in which the behaviour of the net when subjected to various loads is calculated, provided that these loads are represented by means of concentrated forces in nodes.

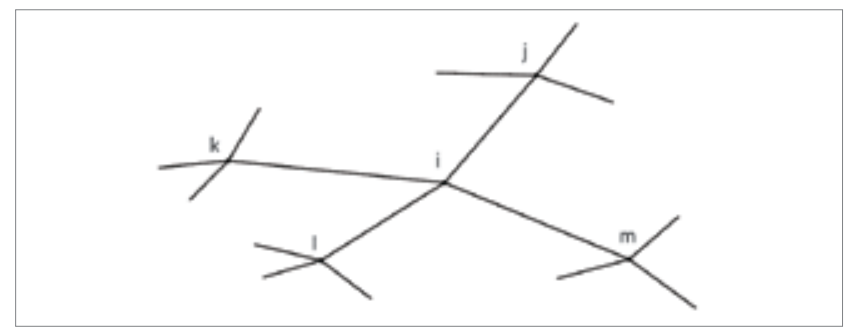

Figure 2. Node $i$, attached elements and neighbouring nodes

In addition to internal nodes in which cables cross, the net will also have support nodes in which cables are linked with "rigid" boundary and, less often, internal linear and point supports. The term "rigid" supports means "traditional" structural elements such as beams, arches, "masts", and "anchors" which, evidently, are not absolutely rigid, but nodes on them can be considered to be fixed. Positions of support nodes are defined in advance - in the computational model these are geometrical boundary conditions. One of the ways in which designers can influence the net form is to change the arrangement and form of fixed supports or, in the model, of geometrical boundary conditions. At that, changed boundary conditions are the input data for the repeated form finding procedure.

As positions of internal nodes are the unknowns in the form finding phase, these nodes will also be referred to as free nodes. The set of free node labels will be denoted by $N_{f}$ and the set of support node labels by $N_{s}$. The number of free nodes is $n_{f}=$ $\operatorname{card} N_{f}$ while the number of support nodes is $n_{s}=\operatorname{card} N_{s}$. The following is valid for the set of all node labels $N=N_{f} \cup N_{s^{\prime}}$ as $N_{f}$ $\cap N_{s}=\varnothing$ and this gives $n=\operatorname{card} N=n_{f}+n_{s}$.

The element between the nodes $i$ and $j$ will be denoted by $(i, j)$, and the set of labels of all elements by $B$; the number of elements will be $n_{b}=$ cardB. The nodes that are linked with elements by the node $i$ will be called "neighbours" to node $i$, and the set of their labels will be denoted by $N_{i}$.

The net topology describes the connection between elements and nodes: elements "belonging" to a cable, elements attached to a node, "neighbours" to a node, etc. The net topology is defined in advance.

The geometry of the net form is a crucial parameter for form finding. The net form must meet structural, functional and aesthetic requirements. In the computational model, it is defined by nodal coordinates. As the required net form is an equilibrium configuration of prestressing tensile forces in the system formed of hinged bars, coordinates of free nodes are basic unknowns in the form finding procedure. In some cases it is appropriate, and even necessary, to introduce, in addition to force equilibrium requirement in nodes, some additional requirements expressed in form of kinematic constraints that link unknown coordinates.

The values of prestressing tensile forces in cables or elements can be defined in advance, but may also be considered as unknowns that are determined by node balancing and, perhaps, by meeting additional kinematic requirements. 


\section{Equilibrium of free node $i$}

Nodal equilibrium equations, which are affected solely by prestressing forces in connected elements, form the basis for finding the form of prestressed cable structures.

Three equilibrium equations - equilibrium equations of force projections in connected elements on three coordinate axes can be written for each free node $i$ :

$$
\sum_{j \in \mathcal{N}_{i}} S_{i, j} \cos \alpha_{i, j}=0, \sum_{j \in \mathcal{N}_{i}} S_{i, j} \cos \beta_{i, j}=0, \sum_{j \in \mathcal{N}_{i}} S_{i, j} \cos \gamma_{i, j}=0
$$

where

- $S_{i, j}$ is the force value in the element $(i, j)$, with positive orientation from the node $i$ toward the node $j$,

- $\alpha_{i, j^{\prime}} \beta_{i, s^{\prime}} \gamma_{i, j}$ are angles between coordinate axes and axis of the element $(i, j)$, oriented from $i$ toward $j$,

hence

$\cos \alpha_{i, j}=\frac{x_{j}-x_{i}}{\ell_{i, j}}, \cos \beta_{i, j}=\frac{y_{j}-y_{i}}{\ell_{i, j}}, \cos \gamma_{i, j}=\frac{z_{j}-z_{i}}{\ell_{i, j}}$

where

$$
\ell_{i, j}=\ell\left(x_{i}, y_{i}, z_{i}, x_{j}, y_{j}, z_{j}\right)=\sqrt{\left(x_{j}-x_{i}\right)^{2}+\left(y_{j}-y_{i}\right)^{2}+\left(z_{j}-z_{i}\right)^{2}}
$$

is length of the element $(i$,$) . If (2)$ and $(3)$ are inserted into (1), the nonlinear algebraic equation system will be obtained

$$
\begin{aligned}
& \sum_{j \in \mathcal{N}_{i}} S_{i, j} \frac{x_{j}-x_{i}}{\sqrt{\left(x_{j}-x_{i}\right)^{2}+\left(y_{j}-y_{i}\right)^{2}+\left(z_{j}-z_{i}\right)^{2}}}=0, \\
& \sum_{j \in \mathcal{N}_{i}} S_{i, j} \frac{y_{j}-y_{i}}{\sqrt{\left(x_{j}-x_{i}\right)^{2}+\left(y_{j}-y_{i}\right)^{2}+\left(z_{j}-z_{i}\right)^{2}}}=0, \\
& \sum_{j \in \mathcal{N}_{i}} S_{i, j} \frac{z_{j}-z_{i}}{\sqrt{\left(x_{j}-x_{i}\right)^{2}+\left(y_{j}-y_{i}\right)^{2}+\left(z_{j}-z_{i}\right)^{2}}}=0
\end{aligned}
$$

for $i \in \mathrm{N}_{\mathrm{f}}$. Thus the system contains $3 n_{f}$ equations.

Any set of $n_{f}$ coordinate triples $\left(x_{i}, y_{i}, z\right), i \in N_{f}$ and $n_{b}$ force values $S_{i, j^{\prime}}(i, j) \in B$, which is compliant with these equations forms an equilibrium configuration. Equations of the system (4) do not contain functions or coefficients (such as, for instance, coefficients of the stiffness matrix in the displacement method) that express the constitutive relation between cable extensions and force values in cables. Therefore, the equilibrium configuration of the net is independent of the cable elasticity moduli and cross-sectional areas of cables. Thus the form finding problem is a static problem, and its solution is a "pure, non-materialised figure of equilibrium" [6].

\section{Minimal cable net}

The total length of cables within a net is expressed with the function $\mathfrak{L}$, which is defined with the following expression:

$$
\mathcal{L}\left(\left\{x_{k}, y_{k}, z_{k}\right\}_{k \in \mathcal{N}_{f}}\right)=\sum_{(i, j) \in \mathcal{B}} \ell_{i, j}
$$

Although the expression for the total cable length includes coordinates of all nodes, the coordinates of support nodes are known, and so $L$ is the function of free node coordinates, $\mathfrak{L}$ : $\mathbb{R}^{3 n f} \rightarrow \mathbb{R}$. If we equate its derivatives with respect to free node coordinates to zero, we obtain the system of $3 n_{f}$ equations with $3 n_{f}$ unknowns:

$$
\frac{\partial \mathcal{L}\left(\left\{x_{k}, y_{k}, z_{k}\right\}_{k \in N_{f}}\right)}{\partial x_{i}}=0, \frac{\partial \mathcal{L}\left(\left\{x_{k}, y_{k}, z_{k}\right\}_{k \in N_{f}}\right)}{\partial y_{i}}=0, \frac{\partial \mathcal{L}\left(\left\{x_{k}, y_{k}, z_{k}\right\}_{k \in N_{f}}\right)}{\partial z_{i}}=0
$$

for $i \in \mathrm{N}_{\mathrm{f}}$ Its solution are free node coordinates for which the function $\mathfrak{L}$ assumes minimal value. The expression for derivative of the function $\mathfrak{L}$ with respect to one of coordinates of the node $i$ will contain only addends which contain in their numerator the same coordinate of neighbouring nodes. For instance,

$\frac{\partial \mathcal{L}\left(\left\{x_{k}, y_{k}, z_{k}\right\}_{k \in \mathcal{N}_{f}}\right)}{\partial x_{i}}=-\sum_{j \in \mathcal{N}_{i}} \frac{x_{j}-x_{i}}{\ell_{i, j}}=0$

The solution of the system can also be understood as root of vector function of a vector variable,

$f\left(x_{f}\right)=0$,

where $f: \mathbb{R}^{3 n f} \rightarrow \mathbb{R}^{3 n f}$, and coordinates of free nodes are lined up in the vector $x_{f} \in \mathbb{R}^{3 n f}$. If $S$ is the constant, $S \neq 0$, then we have

$$
\frac{\partial\left(S \mathcal{L}\left(\left\{x_{k}, y_{k}, z_{k}\right\}_{k \in N_{f}}\right)\right)}{\partial x_{i}}=S \frac{\partial \mathcal{L}\left(\left\{x_{k}, y_{k}, z_{k}\right\}_{k \in \mathcal{N}_{f}}\right)}{\partial x_{i}} \quad \text { etc. }
$$

which means that the "position" of the minimum (point in space $\mathrm{R}^{3 n f}$ ) does not change; the only thing that changes is its value. Consequently, if force values in all elements $(i, j)$ are

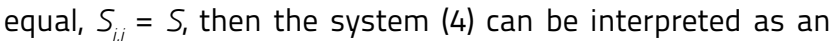
expression of requirements of minimal total cable-length: free node coordinates, obtained as solution of the system, provide the form of the net for which the sum of cable lengths is smaller than the sum of cable lengths in any other form that the net can assume. This form of the net is called the minimal or geodetic cable net; as already shown, it does not depend on the value of force $\mathrm{S}$ from the formal mathematical standpoint, but the force has to be tensile for a physically achievable solution, $\mathrm{S}>0$.

Minimal nets over the plan-view area $[0,1] \times[0,1]$, with boundary nodes whose coordinates comply with the equation $z=1 / 2 x y$, are presented in Figures 3.a) and b); net cables in Figure a) are generatrices of a hyperbolic paraboloid. Minimal nets presented in Figures 3.c) and d) are spread over the planview area $[-1,1] \times[-1,1]$. Coordinates of their boundary nodes are compliant with the equation $z=\ln (\cos x / \cos y)$.

Examples from Figure 3 have been solved using the program function newton_krylov, contained in the program package 

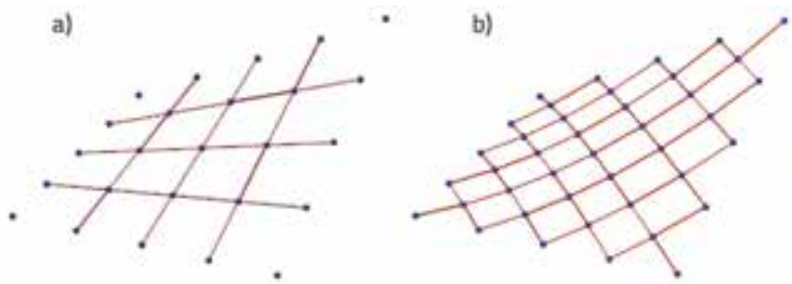

Figure 3. Minimal nets

SciPy [12] which is, together with many others, included in the symbolic program package Sage $[13,14]$. This function is the implementation of the Newton-Krylov procedure for solving the nonlinear equation systems, i.e. for finding the root of the vector function of the vector variable $[15,16]$. The NewtonKrylov procedure is a variant of the known Newton-Raphson procedure [15, 17]. In every step of the external NewtonRaphson loop, linear systems

$\mathbf{f}\left(\mathbf{x}^{(k)}\right)+\mathbf{J}^{(k)}\left(\mathbf{x}-\mathbf{x}^{(k)}\right)=0$

are solved by the selected Krylov iteration procedure (internal loop) and, at that, the products of the Jacobian matrix $J(k)=$ $\nabla \mathbf{f}\left(\mathbf{x}^{(\mathrm{k})}\right)$ and vector $\mathbf{v}$ are calculated directly, without calculation of the matrix itself, in such a way that they are approximated by numerical derivation

$\mathbf{J}^{(k)} \mathbf{V} \approx\left[\mathbf{f}\left(\mathbf{x}^{(k)}+\varepsilon \mathbf{v}\right)-\mathbf{f}\left(\mathbf{x}^{(k)}\right)\right] / \varepsilon$

where $\varepsilon$ is a small number, somewhat greater that the root of mechanical precision of the real number representation $\varepsilon_{\text {mach. }}$. The following Krylov procedures can be used in the newton_ krylov function for solving linear equation systems: Conjugate Gradient Squared (cgs), BIConjugate Gradient STABilized (bicgstab), MINimum RESidual (minres), Generalized Minimal RESidual (gmres) [15] i "Loose" GMRES (Igmres) [18].

The global convergence in the newton_krylov function is ensured by line search. At that, the Armijo criterion without an additional curvature criterion or with this criterion (Wolfe criteria) is used for the step length determination [15].

Symbolic capabilities of the Sage program are used for preparation of the vector function of the vector variable (introduction of a set of symbolic variables for node coordinates, definition of the functions $\boldsymbol{l}_{\mathrm{i}, \mathrm{j}}$ and $\mathfrak{L}$, differentiation of the function $\mathfrak{L}$ with respect to its variables), which is the input parameter for the newton_krylov function.

The data about duration of the net form finding calculations from Figure 3.b) are shown in Table 1. The net has 25 free nodes, which means that the system contains 75 equations with 75 unknowns. For initial approximation, it was assumed that all free nodes lie in the plane $z=0$ forming a regular net. The criterion for interruption of the iteration procedure was $\left\|r^{(k)}\right\|_{\infty} \leq 6 \cdot 10^{-6}$, where $r^{(k)}=f\left(x_{f}^{(k)}\right)$ is the residual.

To enable minimal configuration of the net, the sliding of cables one over another must not be prevented during the prestressing procedure. Only after the final minimal form has
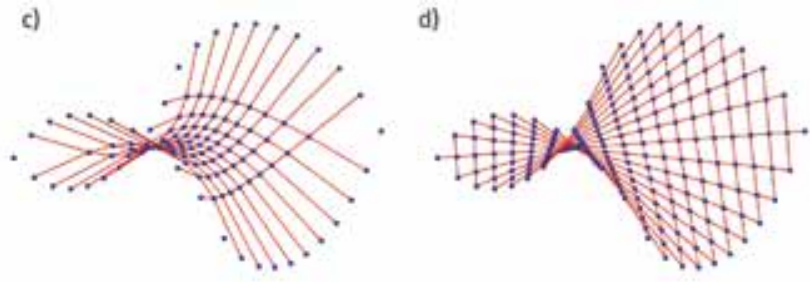

been achieved, cables are connected in points in which they cross one another, so as to increase stiffness of the net and to prevent additional sliding of cables after application of load. Several cable connection possibilities are presented in Figure 4. (to avoid the use of great-diameter cables, larger nets are often realized with two parallel cables of smaller diameter, Figures 4.c i 4.d).

Table 1. Duration of calculations using variants of the Newton-Krylov procedure

\begin{tabular}{|c|c|c|c|}
\hline $\begin{array}{c}\text { Linear system } \\
\text { solving procedure }\end{array}$ & $\begin{array}{c}\text { Line } \\
\text { search }\end{array}$ & $\begin{array}{c}\text { Number of } \\
\text { external loop } \\
\text { steps }\end{array}$ & $\begin{array}{c}\text { Duration of } \\
\text { calculation }\end{array}$ \\
\hline cgs & armijo & 5 & $45,21 \mathrm{~s}$ \\
\hline cgs & wolfe & 4 & $44,22 \mathrm{~s}$ \\
\hline bicgstab & armijo & 5 & $36,98 \mathrm{~s}$ \\
\hline bicgstab & wolfe & 4 & $36,23 \mathrm{~s}$ \\
\hline minres & armijo & 5 & $22,24 \mathrm{~s}$ \\
\hline minres & wolfe & 4 & $25,16 \mathrm{~s}$ \\
\hline gmres & armijo & 5 & $27,21 \mathrm{~s}$ \\
\hline gmres & wolfe & 4 & $28,62 \mathrm{~s}$ \\
\hline Igmres & armijo & 5 & $40,08 \mathrm{~s}$ \\
\hline Igmres & wolfe & 4 & $36,32 \mathrm{~s}$ \\
\hline
\end{tabular}
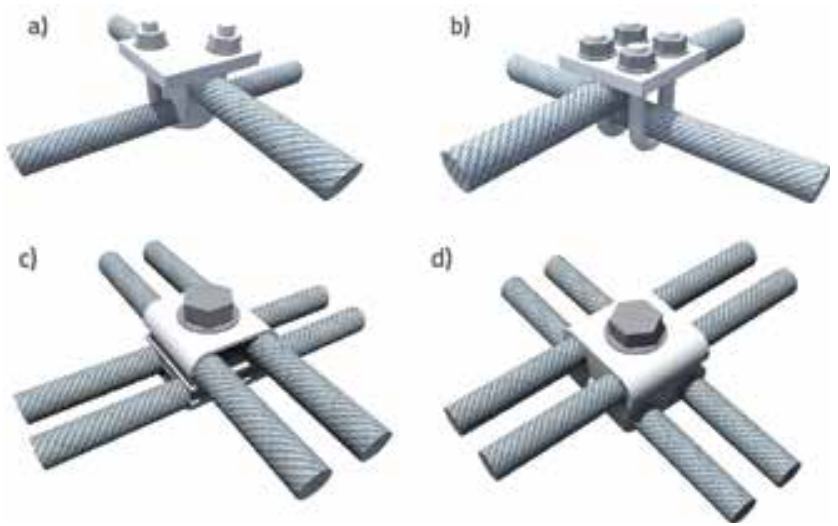

Figure 4. Cable connections (redrawn from [19])

\section{Generalised minimal cable nets}

It can easily be seen that for different force values $S_{i, j}$ the equilibrium equation system (4) expresses requirements for minimum of function $\mathfrak{E}$ given by the expression 
$\mathfrak{E}\left(\left\{x_{k}, y_{k}, z_{k}\right\}_{k \in \mathcal{N}_{f}}\right)=\sum_{(i, j) \in \mathcal{B}} S_{i, j} \ell_{i, j}$

If two cables are connected in the node before the force values along them are equalised, then the connection must transfer from one cable to the other not only the normal contact force but also the tangential force (which prevents cables from sliding). As one cable passes above or under the other cable, their axes do not intersect (they only intersect in our computational model). As force values of two elements connected in a node are not equal, $S_{i, j} \neq S_{i, k}$ the moments of these forces with respect to the axis of the transverse cable will also not be equal: e $S_{i, j} \neq e S_{i, k}$ (Figure 5.a). The node will rotate so as to reduce the arm of the greater force and balance the moments: $e_{i, j} S_{i, j}=e_{i, k} S_{i, k}$ (Figure 5.b). Differences in force values in elements of a cable should be as small as possible, so as to reduce the node rotation.

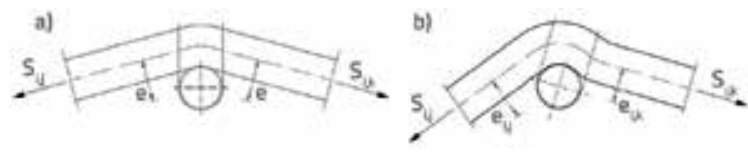

Figure 5. Distortion of cable connection

If different cables are tensioned with different forces and are linked in nodes only after they slide into the equilibrium configuration, force values $S_{i, j}$ will be equal along individual cables, and so the nodes will not be distorted. Such nets, with different force values in different cables, but with unchangeable forces along cables, will be called "natural" generalised minimal cable nets (because a "net comes into a natural equilibrium configuration if sliding is enabled during prestressing", [10]), unlike the "abstract" generalisation in which force values $S_{i, j}$ are completely independent from one another. It should however be noted that, after connection of cables, force values in nodes will change by different amounts under variable loads, and so they will not be equal along individual cables even in "natural" nets. Nevertheless, these differences will be small compared to prestressing force values.
The possibility of assigning different force values in different cables greatly increases the set of forms achievable in forming prestressed cable structures; at that, proportions of forces in cables are significant, rather than their "absolute" values. If forces in other cables do not change, the selected cable is tensioned due to increase in force, and the total length of elements of which it is formed reduces and, at that, the spatial polygonal line formed by these elements is straightened and becomes close to rectilinear connection at its ends (Figure 6, compare with Figure 3.b, although a cable is a polygonal line rather than a curve, it is often said that its curvature decreases by tensioning.
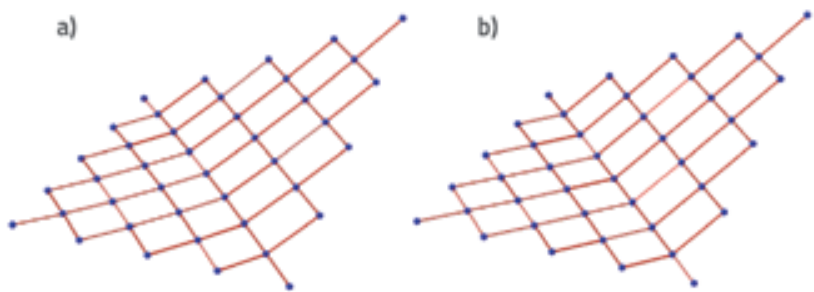

Figure 6. Force in one cable: a) 10 times greater; b) 50 times greater than the force in other cables

Cable nets in examples shown in Figures 3 and 6 have "rigid" edges (e.g. beams), and so coordinates of all edge nodes are known. Prestressed structures made of cables are often made with edge cables. These edge cables are connected with "rigid" structural elements only is some points, usually in terminal ones, and so only the coordinates of these "support" nodes are known. For that reason, form finding of edge cables becomes a part of form finding of the entire net. Force values are much greater in edge cables compared to internal cables.

However, as can be seen from examples given in Figure 7, it may happen that two or more nodes "slide" into a single point along the edge cable. This will happen regardless of force values in edge cables - even by considerably increasing these forces compared to forces in connected cables it would not be possible to prevent the unconfined approaching and merging

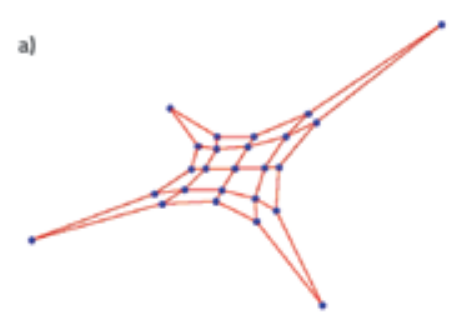

d)

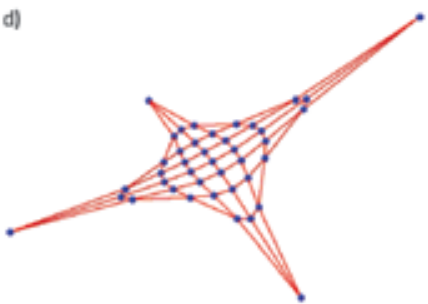

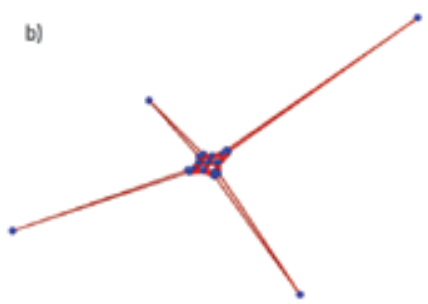

e)

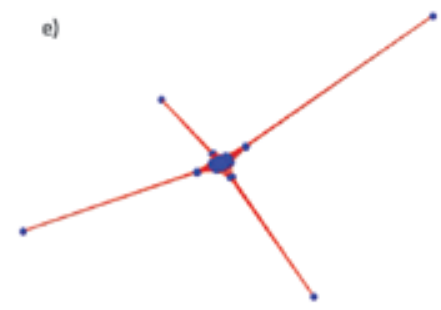

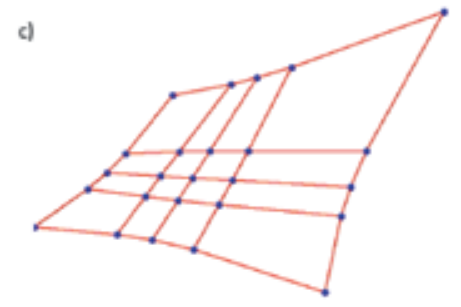

f)

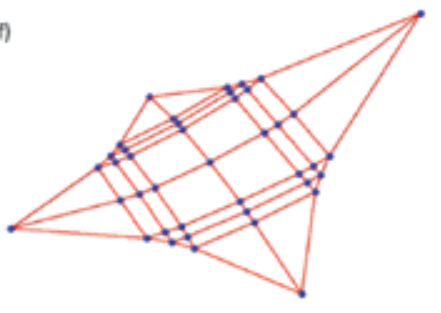

Figure 7. Approaching of nodes: a), c) and b), d) forces in all cables are equal, e) and f) forces in edge cables 10 times greater than those in internal cables 
of nodes. The approaching of nodes can however be "slowed down" by increasing force values in edge cables (Figures 7.a and 7.b compared with 7.c, and 7.d and 7.e compared with 7.f), but nontrivial "non-singular" balanced state does not exist.

Other than being senseless from the net forming standpoint, the node merging causes problems in numerical solution of equilibrium equation systems (4). If two nodes fall into a single point, the length of the element in between them is equal to zero, and differences in individual node coordinates are also equal to zero, and so after insertion in equilibrium equations - where in addends we have differences in coordinates in numerators, and lengths are given in denominators - the corresponding addends are indeterminate expressions $0 / 0$, which causes interruption of the computer program, most often accompanied with an incomprehensible error message. This is why sliding will be prevented in points where internal cables are connected with edge cable (the connection is presented in Figure 8), while the length of elements at edge cables will be set by kinematic constraints:

$$
\ell_{i, j}=\bar{\ell}_{i, j}
$$

for $(i, j) \in \mathcal{B}_{\text {c }}$ the set of elements with defined lengths is denoted by $\mathcal{B}_{c^{\prime \prime}}$ and their number by $n_{c^{\prime}} n_{c}=\operatorname{card} \mathcal{B}_{c^{\prime}}$.

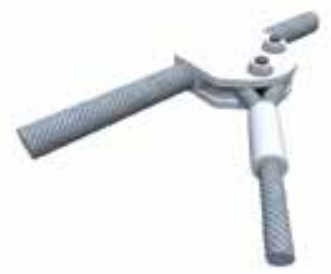

Figure 8. Internal cable and edge cable connection (redrawn from [19])

Kinematic constraints will be introduced in the computation by means of Lagrange multipliers [20]. Constraint equations will therefore be written as follows:

$\ell_{i, j}-\bar{\ell}_{i, j}=0$

where the left side will be multiplied by newly introduced variables $\lambda_{\mathrm{i}, j^{\prime}}$ called Lagrange multipliers, which will be added to the function $\mathfrak{E}$. The new function $\mathfrak{E}_{c}: \mathrm{R}^{3 \mathrm{nf}+\mathrm{nc}} \rightarrow \mathrm{R}$ will thus be obtained. It is defined with the expression:

$\mathfrak{E}_{c}\left(\left\{x_{k}, y_{k}, z_{k}\right\}_{k \in N_{f}},\{\lambda\}_{(i, j) \in \mathcal{B}_{c}}\right)=\sum_{(i, j) \in \mathcal{B}} S_{i, j} \ell_{i, j}+\sum_{(i, j) \in \mathcal{B}_{c}} \lambda_{i, j}\left(\ell_{i, j}-\overline{\ell_{i, j}}\right)$

After derivatives of the function $\mathfrak{E}_{c}$ with respect to coordinates of free nodes are equated to zero, the following equations are obtained (after multiplication with -1 )

$\sum_{j \in N_{i}} S_{i, j} \frac{x_{j}-x_{i}}{\ell_{i, j}}+\sum_{j \in N_{i}^{i}} \lambda_{i, j} \frac{x_{j}-x_{i}}{\ell_{i, j}}=0$
$\sum_{j \in N_{i}} S_{i, j} \frac{y_{j}-y_{i}}{\ell_{i, j}}+\sum_{j \in N_{i}^{i}} \lambda_{i, j} \frac{y_{j}-y_{i}}{\ell_{i, j}}=0$
$\sum_{j \in N_{i}} S_{i, j} \frac{z_{j}-z_{i}}{\ell_{i, j}}+\sum_{j \in N_{i}^{i}} \lambda_{i, j} \frac{z_{j}-z_{i}}{\ell_{i, j}}=0$ for $i \in N_{a \prime}$ where $N_{i}^{c}$ is the set of labels of "neighbours" to the node $i$ which are connected with this node by elements of a defined length. The equating with zero of derivatives of the function $\mathfrak{E}_{c}$ with respect to the Lagrange multipliers results in kinematic constraint equations (8). Equations (10) and (8) together form the system containing $3 n_{f}+n_{c}$ equations with $3 n_{f}+n_{c}$ unknowns; now, the unknowns are not only coordinates of free nodes: the multipliers $\lambda_{i, j}$ are also regarded as unknowns. Equations (10) can also be written as:

$\sum_{j \in \mathcal{N}_{i} \backslash \mathcal{N}_{i}^{c}} S_{i, j} \frac{x_{j}-x_{i}}{\ell_{i, j}}+\sum_{j \in \mathcal{N}_{i}^{c}}\left(S_{i, j}+\lambda_{i, j}\right) \frac{x_{j}-x_{i}}{\ell_{i, j}}=0 \quad$ etc.

from which it can be seen that multipliers $\lambda_{i, j}$ are additional force values that are needed to provide for the required lengths of the elements. This means that we no longer have a full "control" over forces in cables - force values defined in edge cables will be adapted by $\lambda_{i, j}$ values that are not known before the system has been solved. And, as could have been expected because we have prevented the sliding, the values of forces in elements of the edge cable will not be equal to one another.

Figure 9 shows two nets with the defined length of edge cable elements. Edge element lengths were selected by means of average element-length values on individual edge cables as determined after five steps of the force density iteration method (Section 8) with allowed sliding of all cables. In all internal elements the forces amount to 1 . Initial force values in edge cable elements amounted to 10 . The obtained values of $\lambda_{i, j}$ range from -0.524 to 0.309 , which means that final force values in edge cables range from 9.476 to 10.309 .
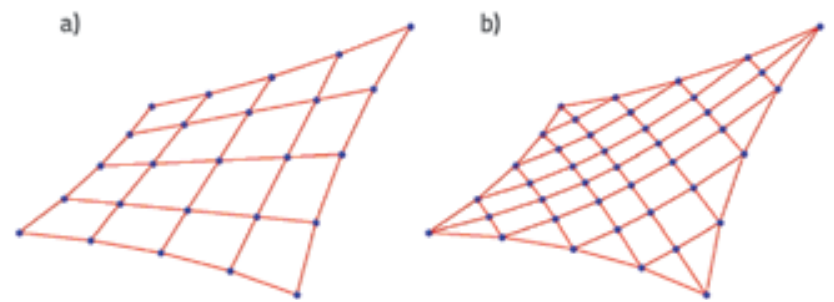

Figure 9. Minimal nets with edge cables, with the defined lengths of sections in between the nodes

The newton_krylov function was also used for solving the equation system (10). The convergence is however disappointingly slow. For instance, 22 external loop steps were needed to find the net form from Figure 9.b using the Armijo criterion in line search and Igmres procedure for solving linear systems, and by applying the criterion for interruption of the iteration procedure $\left\|r^{(k)}\right\|_{\infty} \leq 1 \cdot 10^{-5}$, and the calculation lasted 850 $\mathrm{s}$. The convergence is somewhat faster when the more stringent Wolfe criteria are used: 18 external loop steps in $732 \mathrm{~s}$. This is not due to a greater number of equations and unknowns (compared to Table 1): additional $12 \times 3$ node coordinates at edge cables and 16 multipliers, i.e. additional 36 equilibrium equations and 16 kinematic constraint equations. The convergence is much slower because, as will be shown in the next section, the use of Lagrange 
multipliers changes the problem from the minimisation problem to the saddle point problem.

\section{Steiner's problem}

The simplest example of the spatial net is one free node connected with the base by four elements and, at that, four support nodes are not in a single plane (it can be assumed that in Figure 2 the nodes $j, k$, land $m$ are support nodes and that $i$ is a free node). A natural generalised minimal net will contain two crossed cables. The form finding problem has three unknowns - coordinates of free node.

If the spatial aspect is renounced, a free node can be tied to the base with three elements. If prestressing forces are introduced into elements, the free node will come to the plane that is defined by three support nodes. From the static standpoint, this is due to the fact that the equilibrium of three forces is impossible if they do not lie in the same plane. From the standpoint of minimisation of the total length of elements, it can easily be seen that the sum of lengths of connections of three points in the plane with a point out of plane will be greater than the sum of lengths of connections with orthogonal projection of this point onto the plane.

In plane, the form finding problem, formulated as a minimisation problem, can be expressed in the following way: find the system of straight sections linking points $A, B$ and $C$ with the smallest possible total length. If expressed in this way, the problem carries the name of Jacob Steiner (1796-1863), although the problem was set earlier, i.e. in 1646, by Pierre de Fermat (16011665), and was probably partly solved in that very year by Evangelista Torricelli (1608-1647) [21].

If all angles of the triangle with vertices $A, B$ and $C$ amount to less than $120^{\circ}$, then the unique point $F$, called the Fermat point, for which the sum of segment lengths $A F, B F$ and $C F$ is the smallest possible, is the point within the triangle for which the following applies $\angle A F B=\angle B F C=\angle C F A=120^{\circ}$, (Figure 10.a). The geometric proof of the validity of the solution is given, for instance, in [21]. If we assume that $(A, F),(B, F)$ and $(C, F)$ are tensioned strings, then a simple and brief "static proof" can be proposed: forces are equal to one another in all three cables, $S_{\mathrm{A}, \mathrm{F}}=S_{\mathrm{B}, \mathrm{F}}=S_{\mathrm{C}, \mathrm{F}}=S_{\text {, and so }}$ the equilibrium triangle of forces is equilateral; in an equilateral triangle the angles are at $60^{\circ}$, and their co-angles at $120^{\circ}$ (Figure 10.b). In other words, in the Fermat point is the minimum of the function defined with the expression

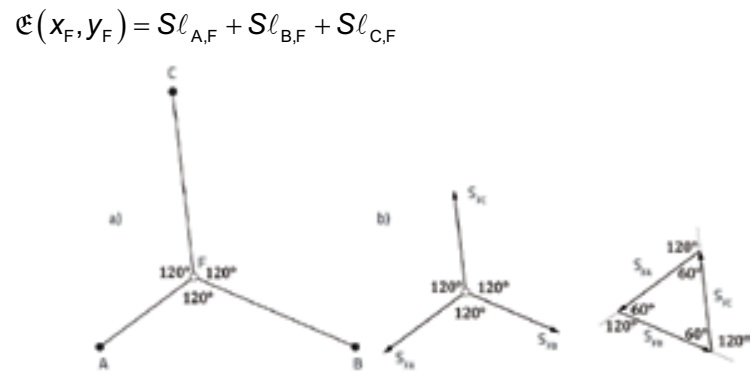

Figure 10. a) Solution to Steiner's problem, b) equilibrium of three forces
One of geometric constructions of the Fermat point is based on the theorem (generalisation of the Thales' theorem) that all inscribed angles of the circle above the same chord, on one of its sides, are equal to each other, and that the sum of two inscribed angles on different sides of the chord amounts to $180^{\circ}$. If we make circles above segments $A C$ and $B C$, whose inscribed angles are $120^{\circ}$ on those sides of these segments where points $B$ and $A$ lie, these circles will intersect, in addition to the point $C$, in the Fermat point $F$. As on opposite sides of the segments their inscribed angles amount to $60^{\circ}$, these circles are easy to construct: we will make above segments $A C$ and $\mathrm{CB}$ equilateral triangles $\triangle \mathrm{ACB}$ and $\triangle \mathrm{CB} \overline{\mathrm{A}}$ with vertices on the sides opposite to those on which inscribed angles must have $120^{\circ}$; the circles sought are the circles through vertices of these triangles (Figure 11). Third circle, circumscribed to equilateral triangle above the segment $A B$ with the vertex $\bar{C}$ on the side opposite to point $C$, will also pass through the point $F$.

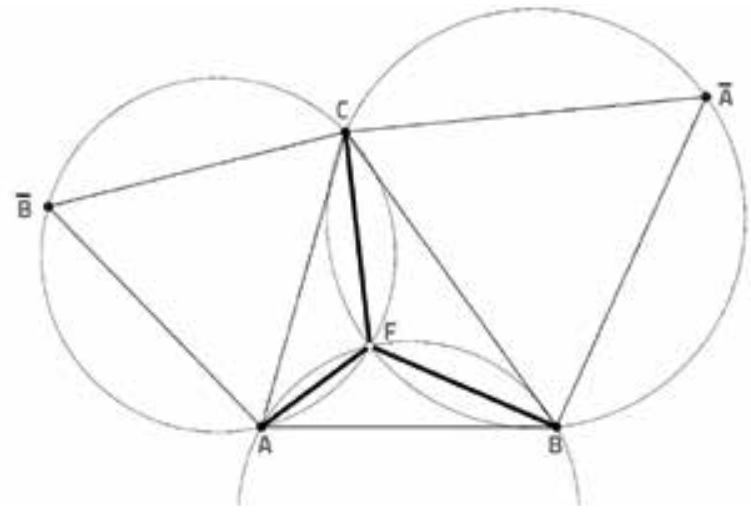

Figure 11. Geometric construction of solution to Steiner's problem

If one angle of the triangle $\triangle A B C$, for instance the angle in the vertex $C$, has $120^{\circ}$ or more, then the shortest link between the points $A, B$ and $C$ is composed of sections $A C$ and $C B$ (this case was defined not earlier than in 1834 [21]). However, if we construct, just like in the first case, circles with the chords $A C$ and $C B$, then their arches lying on the same sides of these chords as the triangles $\triangle A C \bar{B}$ and $\triangle C B \bar{A}$, will intersect, i.e. on the sides on which inscribed angles have $60^{\circ}$ (Figure 12.a)); the obtained intersection will be marked with $G$. Now the angles between connections AG and CG and between connections CG and $\mathrm{BG}$ will have $60^{\circ}$, while the angle between connections $A G$ and $B G$ will have $120^{\circ}$. In static interpretation, the point $G$ is the node which is the meeting point of elements $(A, G),(B$, $\mathrm{G}$ ) and $(C, G)$ in which all forces are of equal intensity, but the forces $S_{G, A}$ and $S_{G, B}$ are tensile while the force $S_{G, C}$ is compressive (Figure 12.b) or vice versa (Figure 12.c). Consequently, the described construct minimises the function $\mathfrak{E}$ given by the expression

$\mathfrak{E}\left(x_{\mathrm{G}}, y_{\mathrm{G}}\right)=S \ell_{\mathrm{A}, \mathrm{G}}+S \ell_{\mathrm{B}, \mathrm{G}}-S \ell_{\mathrm{C}, \mathrm{G}}$

where $S$ is the intensity of force in elements, $S=\left|S_{A, G}\right|=\left|S_{B, G}\right|$ $=\left|S_{C, G}\right|$. 


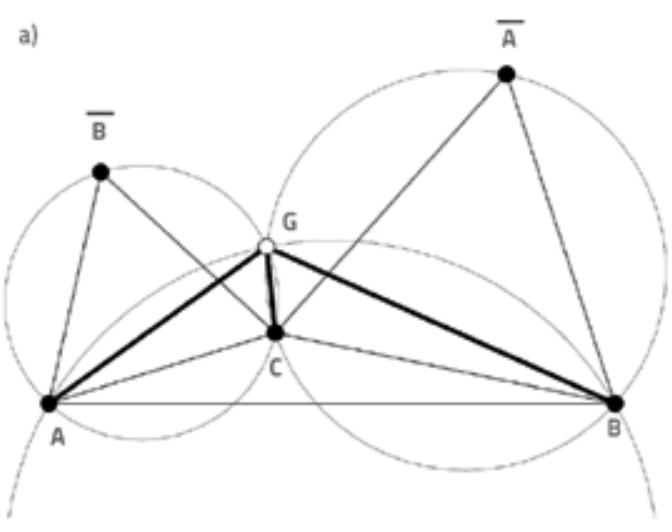

b)

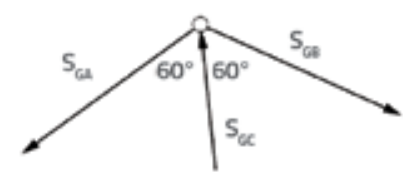

c)

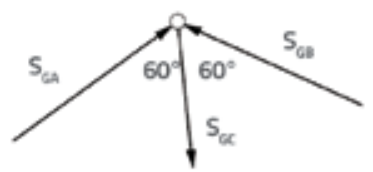

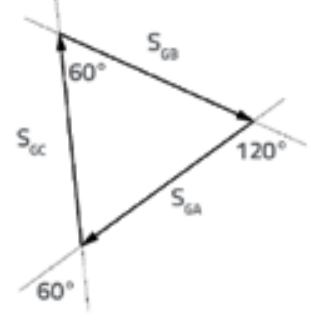

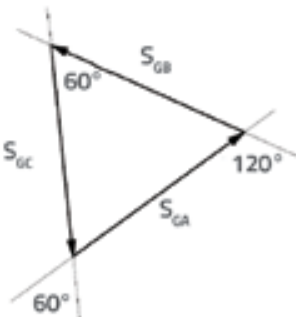

Figure 12. a) Geometric construction of solution to a Steiner's problem variant. b) and c) Systems of tensile and compressive elements

As an example of the Steiner's problem, let the points $A(0,0)$, $B(5,0)$ and $C(1,425 ; 5)$ be given. The angles of the triangle $\triangle A B C$ amount to less than $120^{\circ}$, and so the Fermat point exists within the triangle.

The total length of strings, as a function of coordinates of the point $F$ in which all strings are linked to each other, amounts to

$$
\mathcal{L}:\left(x_{F}, y_{F}\right) \rightarrow \sqrt{x_{F}^{2}+y_{F}^{2}}+\sqrt{\left(x_{F}-5\right)^{2}+y_{F}^{2}}+\sqrt{\left(x_{F}-1,425\right)^{2}+\left(y_{F}-5\right)^{2}}
$$

The graph of the function $\mathfrak{L}$ is the surface $\Gamma_{\mathfrak{L}}$ as presented in Figure 13.a). This surface is convex and so it has the lowest point, while the function $\mathfrak{L}$ has a minimum (a function is called convex if the connection between any two points in its graph is above it or, formally, if the following applies: $f(a \mathbf{x}+(1-a)$ $\mathbf{y}) \leq a f(\mathbf{x})+(1-a) f(\mathbf{y})$.). By equating with zero derivatives of the function $\mathfrak{L}$ with respect to coordinates of the point $F$, the following equation system is obtained

$\frac{x_{F}}{\sqrt{x_{F}^{2}+y_{F}^{2}}}+\frac{x_{F}-5}{\sqrt{\left(x_{F}-5\right)^{2}+y_{F}^{2}}}+\frac{x_{F}-1,425}{\sqrt{\left(x_{F}-1,425\right)^{2}+\left(y_{F}-5\right)^{2}}}=0$
$\frac{y_{F}}{\sqrt{x_{F}^{2}+y_{F}^{2}}}+\frac{y_{F}}{\sqrt{\left(x_{F}-5\right)^{2}+y_{F}^{2}}}+\frac{y_{F}-5}{\sqrt{\left(x_{F}-1,425\right)^{2}+\left(y_{F}-5\right)^{2}}}=0$

a)

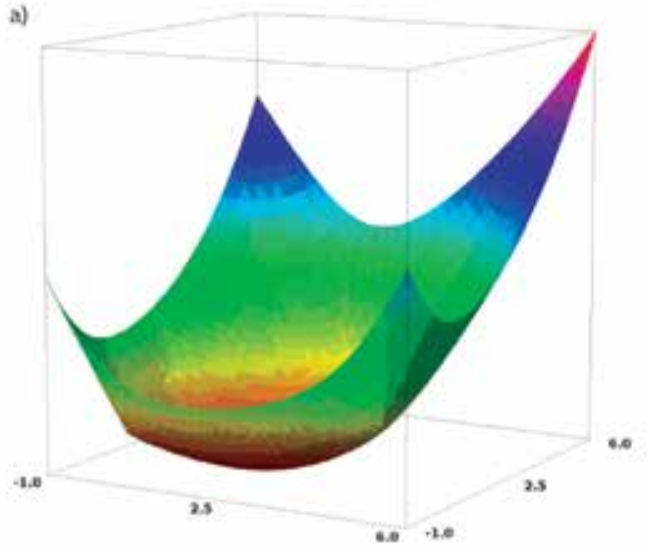

Derivatives of the function $L$ are not defined in points $A, B$ and $C$ : if we insert coordinates of some of them into expressions for derivatives, the numerator and denominator of each addend will be equal to zero in each expression. This inter alia means that the iterative procedure for solving equation systems must not be commenced in any of these points. Similarly, the iteration will fail if we run into any of them by accident during the procedure, and even perhaps if we come close to them (due to numerical errors). The lack of derivatives is reflected on the surface $G_{L}$ by vertices in points $\left(x_{A^{\prime}} y_{A^{\prime}} \mathfrak{L}_{A^{\prime}}\right)$, $\left(x_{B^{\prime}} y_{B^{\prime}} \mathfrak{L}_{B}\right)$ and $\left(x_{C^{\prime}}, y_{C^{\prime}} \mathfrak{L}_{C}\right) ;$ a part around the point $\left(x_{B^{\prime}}, y_{B^{\prime}} \mathfrak{L}_{B}\right)$ is enlarged in Figure 13.b.

The Fermat point is the point $F(1,843503 ; 1,367735)$.

It is known that iterative procedures for solving nonlinear equation systems are sensitive to the choice of the initial approximation of the solution $[22,23]$. As an example, Figure 14 shows numbers of steps in external loop during solution of the system using the function newton_krylov with the solution of the linear system using the gmres method. Each point is coloured with the colour that corresponds to the number of steps needed to reach the solution (with the accuracy of $\left.\left\|r^{(k)}\right\|_{\infty} \leq 1 \cdot 10^{-5}\right)$ if the resolution started in that point; it is

b)

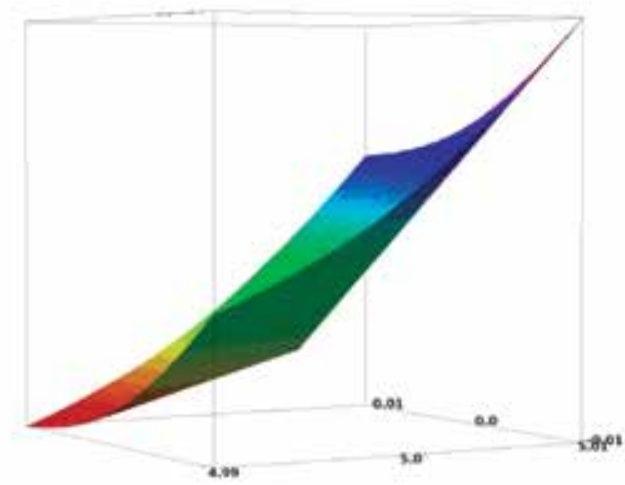

Figure 13. Graph of the function $\mathfrak{L}$ 
highly probable that the solution can not be reached from points marked with the colour denoting the greatest number of steps (we can not make a countless number of steps to determine that the procedure is divergent). The area covered with a regular net containing $101 \times 101$ points was selected in all four cases. The area in the first figure is square $[-5,10]$ $\times[-5,10]$, and so the entire triangle $\triangle A B C$ is included (as we know, we can not start from its vertices, and it would not be advisable to start from their vicinity), while in the remaining three figures the areas measure $5 \times 5,0,2 \times 0,2$ and $0,002 \times$ 0,002 with the centre in point $C$.

We will now set the length of the string $(A, F): \ell_{A, F}=2$ (in the solution to the Steiner's problem it is $\ell_{A, F}=2,295474$ ). By multiplying the introduced kinematic constraint with the Lagrange multiplier $e_{A, F}$ and by adding to the function $\mathfrak{L}$, we obtain the following function

$\mathfrak{E}_{C}:\left(x_{F}, y_{F}, \lambda_{F, A}\right) \rightarrow \sqrt{x_{F}^{2}+y_{F}^{2}}+\sqrt{\left(x_{F}-5\right)^{2}+y_{F}^{2}}+$

$\sqrt{\left(x_{F}-1,425\right)^{2}+\left(y_{F}-5\right)^{2}}+\lambda_{F, A}\left(\sqrt{x_{F}^{2}+y_{F}^{2}}-2\right)$

As this is the function of three variables, its graph is a hypersurface in the four-dimensional space. The cross-section of the hypersurface and hyperplane $x_{F}=1,604358$ is a surface in the three-dimensional space of this hyperplane. In other words, this surface is a graph of the function $\mathfrak{E}_{c}(1,604358 ; ; \cdot ;)$ of two variables $y_{F}$ and $\lambda_{F A}$. As shown in Figure 15. a the axis $y_{F}$ is perpendicular to the plane of the drawing, while the axis $\lambda_{F, A}$ is a horizontal axis parallel to it. Figure 15.b shows the intersection of graph of the function $\mathfrak{E}$ by the hyperplane $y_{F}=1,194167$; now the axis $x_{F}$ is parallel to the plane of the drawing, while the axis $\lambda_{F, A}$ is perpendicular to it. And finally, the graph of the function $\mathfrak{E}_{\mathrm{c}}(\because ; ; 0,117318)$ is presented in

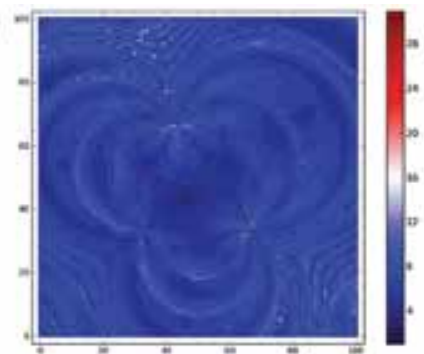

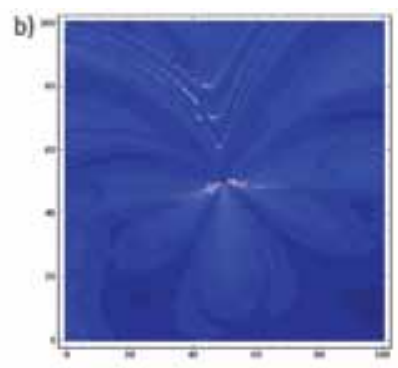

Figure 15.c. This surface is very similar to the graph of the function $\mathfrak{L}$ (Figure 13.a), which is by no means an accident - in fact, $\mathfrak{L}=\mathfrak{E}_{\mathrm{c}}$ $(\cdot,, 0)$. Graphs of all functions $\mathfrak{E}_{\mathrm{c}}\left(\cdot, \cdot, \lambda_{\mathrm{FA}}\right)$ are convex surfaces for selected values of $\lambda_{F, A^{*}}$. However, graphs of the functions $\mathfrak{E}_{\mathrm{C}}\left(X_{\mathrm{F}}\right.$. , .) i $\mathfrak{E}_{\mathrm{C}}\left(\cdot, y_{\mathrm{F}^{\prime}} \cdot\right)$ are not (Figures 15.a and 15.b). The intersections of these surfaces by planes $\lambda_{F, A}=$ const are concave curves, but intersections of the first surface by planes $y_{F}=$ const, and of the second surface by planes $x_{F}=$ const are straight lines (Figures 16.a) and b)). In the lowest point of the convex surface, which corresponds to the minimum of the function whose surface is a graph, the tangential plane is horizontal, and the entire surface is above it. Horizontal tangential planes of surfaces from Figure 15.a and 15.b also intersect these surfaces (e.g. for surface from Figure 15.b, Figure 16.c. The point of tangency of the horizontal tangential plane which touches and intersects the surface is called the saddle point, and the surface itself is called the saddle surface. The surface is partly above and partly below the tangential plane in the saddle point, which means that the saddle point is not the lowest point of the surfaces and that the function, whose graph is this surface, does not have a minimum. The equating of derivatives of the function with zero expresses stationarity conditions, which are minimum conditions for functions with convex graphs only.

As the surfaces $\mathfrak{E}_{\mathrm{C}}\left(x_{F^{\prime}}, \cdot\right)$ and $\mathfrak{E}_{\mathrm{C}}\left(\cdot, y_{\mathrm{F}^{\prime}} \cdot\right)$ do not have minima (although the function $\mathfrak{E}_{\mathrm{c}}\left(\cdot, \cdot, \lambda_{\mathrm{F}, \mathrm{A}}\right)$ has a minimum), the function $\mathfrak{E}_{\mathrm{c}}$ also does not have a minimum. Stationarity conditions for the function $\mathfrak{E}_{\mathrm{C}}$ are

$$
\begin{aligned}
& \frac{\left(1+\lambda_{F, A}\right) x_{F}}{\sqrt{x_{F}^{2}+y_{F}^{2}}}+\frac{x_{F}-5}{\sqrt{\left(x_{F}-5\right)^{2}+y_{F}^{2}}}+\frac{x_{F}-1,425}{\sqrt{\left(x_{F}-1,425\right)^{2}+\left(y_{F}-5\right)^{2}}}=0 \\
& \frac{\left(1+\lambda_{F, A}\right) y_{F}}{\sqrt{x_{F}^{2}+y_{F}^{2}}}+\frac{y_{F}}{\sqrt{\left(x_{F}-5\right)^{2}+y_{F}^{2}}}+\frac{y_{F}-5}{\sqrt{\left(x_{F}-1,425\right)^{2}+\left(y_{F}-5\right)^{2}}}=0 \\
& \sqrt{x_{F}^{2}+y_{F}^{2}}-2=0
\end{aligned}
$$
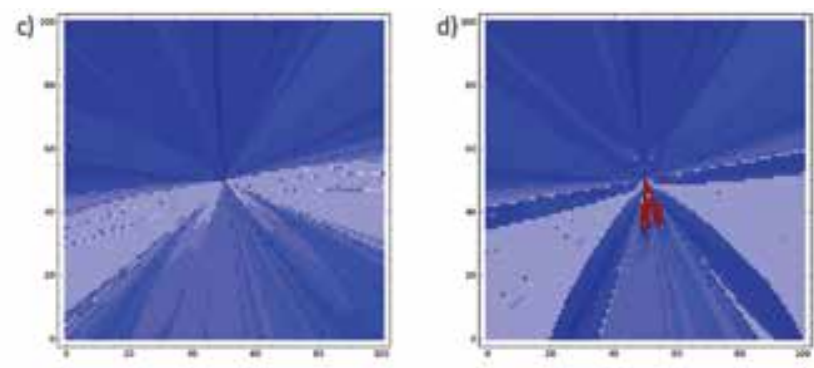

Figure 14. Number of steps until solution of the Steiner's problema

a)

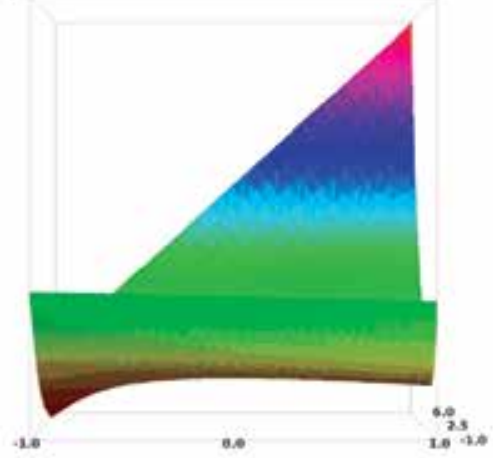

b)

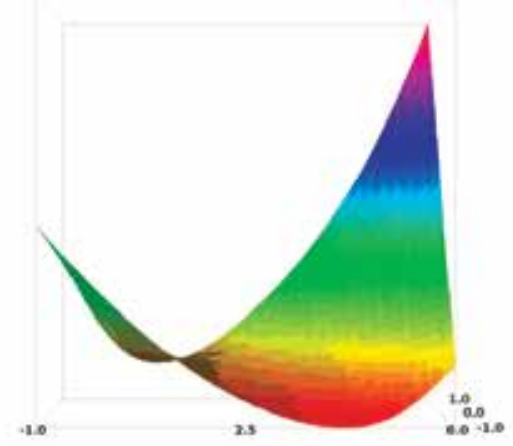

c)

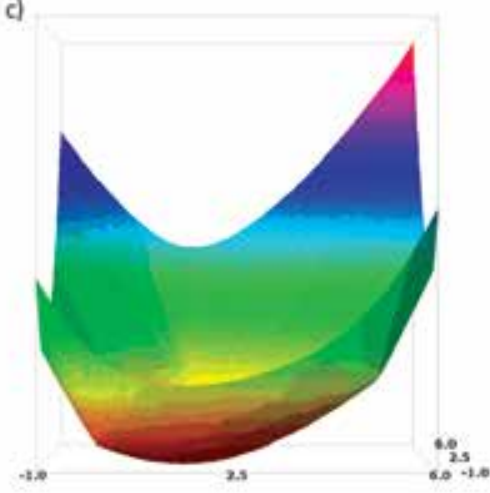

Figure 15. Graphs of functions $\mathfrak{E}_{\mathrm{c}}(1,604358 ; ; \cdot), \mathfrak{E}_{\mathrm{c}}(1,194167 ; \cdot ; \cdot)$ and $\mathfrak{E}_{\mathrm{c}}(\cdot ; \cdot ; 0,117318)$ 
a)

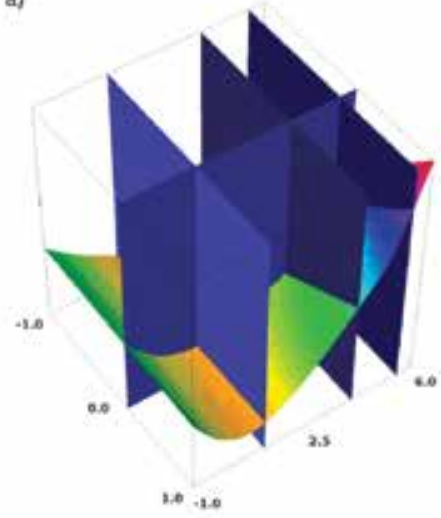

b)

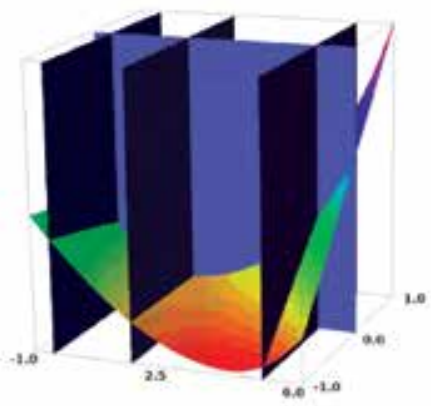

c)

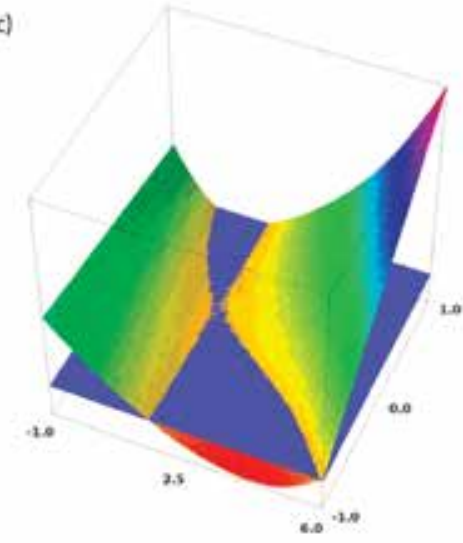

Figure 16. a) i b) Intersections of surfaces $\mathfrak{E}_{\mathrm{c}}(1,604358 ; \cdot ; \cdot)$ and $\mathfrak{E}_{\mathrm{c}}(\cdot ; 1,194167 ; \cdot)$ by vertical planes; $\left.\mathrm{c}\right)$ Tangential plane of the surface $\mathfrak{E}_{\mathrm{c}}(\cdot ;$ $1,194167 ; \cdot)$ in the saddle point

The point $(1,604358 ; 1,194167 ; 0,117318)$ is stationary, and so the $F(1,604358 ; 1,194167)$ and $\lambda_{F, A}=0,117318$. Thus if we wish the string length $(A, F)$ to be $\lambda_{F, A}=2$, the force relationships in strings must be $S_{F, A}: S_{F, B}: S_{F_{1}}=1,117318: 1: 1$.

The problem of finding the saddle point of the function is much more difficult than the problem of finding its minimum. Intuitively, while during finding the minimum of the function the "downward descent" ensures a proper way of getting closer to the solution, in the problem of finding the saddle point this can be a descent to the Dante's ninth circle of hell, i.e. to - $-\infty$. Jacobian matrices have a special structure in problems involving saddle points. Kinematic constraint equations do not contain Lagrange multipliers, and so derivatives of their left sides with respect to multipliers are zero. Consequently, if kinematic constraint equations are lined up after equilibrium equations, and Lagrange multipliers, as unknowns, after free node coordinates, the bottom right block of the Jacobian matrix will be a quadratic zero-matrix of type $n_{c} \times n_{c}$. In our example, the $I_{F, A}$ is the third unknown, and the kinematic constraint equation is the third equation in the system that expresses stationarity conditions, and so the Jacobian matrix, as calculated at the point $(1 ; 1 ; 0.1)$, is

$\mathbf{J}=\left[\begin{array}{ccc}0,649001 & -0,357960 & 0,707107 \\ -0,357960 & 0,619953 & 0,707107 \\ 0,707107 & 0,707107 & 0\end{array}\right]$

This matrix is not positive definite, which limits the number of methods by which the linear system can be solved (the applicable methods are gmres and Igmres).

Figure 17 shows the number of steps that are needed to solve the equation system using the newton_krylov function and the gmres method. As the system now has three unknowns, initial values of the two of them were varied. In the Figures 17.a and 17.b, coordinates of the initial point have changed in the same area as in Figure 14.a. At that, the initial value of the Lagrange multiplier in Figure 17.a amounted to $\lambda_{\text {F.A }}^{(0)}$ $=0,117318$, while in Figure 17.b it was $\lambda_{F, A}^{(0)}=1$. Comparison with Figure 14.a reveals that the convergence is now slower.
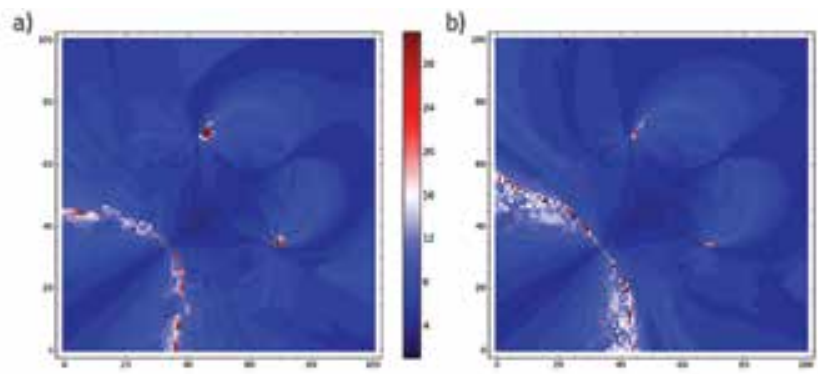

Figure 17. Number of steps until solution of the saddle point problem

\section{Force density method}

If the relationships $S_{i, j} / e_{i, j}$ in the equilibrium system (4) are denoted $q_{i, j^{\prime}}$ the system becomes

$\sum_{j \in \mathcal{N}_{i}} q_{i, j}\left(x_{j}-x_{i}\right)=0, \sum_{j \in \mathcal{N}_{i}} q_{i, j}\left(y_{j}-y_{i}\right)=0, \sum_{j \in \mathcal{N}_{i}} q_{i, j}\left(z_{j}-z_{i}\right)=0$

for $i \in \mathrm{N}_{\mathrm{f}}$. Values

$q_{i, j}=S_{i, j} / \ell_{i, j}$

are called force densities.

Force densities $q_{i, j}$ can be set instead of force values $S_{i, j}$ Therefore, force values in elements and node coordinates are unknowns (total of $n_{b}+3 n_{\mathrm{f}}$ unknowns), but the unknowns are linked with additional $n_{b}$ equations (12). However, additional equations do not complicate the solution of the system. On the contrary: in equations (11) $q_{i j}$ are constant coefficients, and so it can easily be seen that the equilibrium equation system has been "broken down" into three separate systems of $n_{f}$ linear algebraic equations each with $n_{f}$ unknowns $\left\{x_{k}\right\}_{\mathrm{k} \in \mathrm{Nf}}\left\{y_{k}\right\}_{\mathrm{k} \in \mathrm{Nf}} \mathrm{i}$ $\{z\}_{\mathrm{k} \in \mathrm{Nf}}$. These systems are not homogeneous as coordinates of support nodes are known, and so $q_{i, j} x_{j} q_{i, j} y_{j}, q_{i, j} z_{j}$ are free members for $\in N_{\mathrm{s}^{\prime}}$ and they are different from zero, at least in some equations. If all equations are multiplied by the same number, the solution to the system will not be changed, which means that it only depends on force density ratios in elements. 
As coordinates of free nodes are obtained as the solution of the system, the expression (3) can be used to calculate the lengths $e_{i, j}$ of all elements, and then the forces in these elements because, according to (12), they are $\operatorname{arj}_{i, j}=q_{i, j} e_{i, j}$

For sake of completeness, and in accordance with presentations given in sections 4 and 5 , we should also mention variationalformulation of the force density method: H.-J. Schek has already noted in [5] that force density method equations (11) express conditions for minimum of a function

$$
\mathfrak{D}\left(\left\{x_{k}, y_{k}, z_{k}\right\}_{k \in \mathcal{N}_{f}}\right)=\frac{1}{2} \sum_{(i, j) \in \mathcal{B}} q_{i, j} \ell_{i, j}^{2}
$$

and so for equal force densities in all elements, $q_{i, j}=q$, the solution to these equation is given by the net form for which the sum of the cable length squares is a minimum value.

As the systems (11) are linear, they can also be solved by a direct procedure such as the Gaussian elimination; in this case, initial approximate coordinates of free nodes (approximate net form) need not be assumed.

Each solution obtained by the force density method, for any distribution of force densities along elements of the net of a given topology, is an equilibrium configuration. The force density distribution should be selected (among $\infty^{n b}$ of possible ones) in such a way that the final net form is compliant with structural and architectural requirements. However, the term force density is an "artificial" term, derived from formal manipulation of mathematical expressions. The form of the net, and distribution of prestressing forces in the net, must be assumed based on the ratio of two values - one static and the other geometric. One of the coauthors of the method proposes in paper [6], based on "many investigations and practical experiments [...] very simple, rather trivially prescribed force densities are sufficient to generate figures of equilibrium serving as a start in the formfinding process". Lets assume that the net of a given topology is spread out on the ground between supports. Unit force densities are assigned to elements in the internal area of the network, where they are of approximately equal length and, in boundary areas, often irregular in shape, where the lengths of elements that are connected to supports or edge cables are notably different, the elements should be assigned force densities that are inversely proportional to their lengths (at that the element length in the interior is taken to be a unit value). This recommendation can in fact be complemented with an intuitive explanation. If cable length ratios in equilibrium configuration are not significantly altered with respect to the ratios in the initial, "spread out" configuration, then force values in elements will be approximately the same and, even if greater
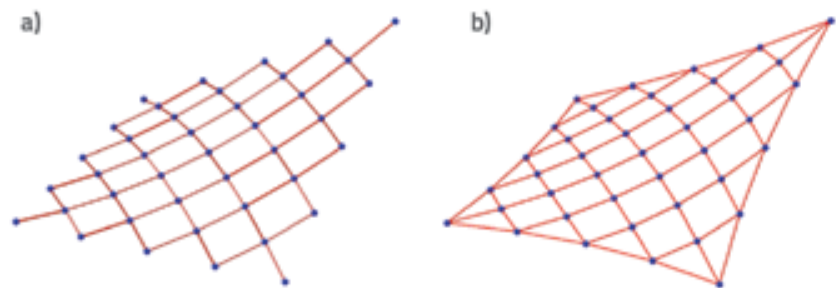

differences are noted, it may be expected that force values along cables will change only gradually, in smaller increments, so that the rotation of nodes (Figure 5) will not be great.

Two networks defined by the force density method are presented in Figures 18.a and 18.b; the first one is the net of equal topology and of equal boundary conditions ("rigid" supports along edges) as a minimal net in Figure 3.b, while the topology and boundary conditions (edge cables, with "rigid" supports in corner nodes only) of the second net are similar to those given for the net shown in Figure 9.b. In the first case all elements are assigned unit force densities and, in the second case, force densities in edge cables are ten times greater that densities in internal ones; as both planview areas are regular, it was not necessary to attribute some other force densities to internal elements linked with supports or to edge cables. In the net shown in Figure 18.b the greatest and the smallest force values in internal cables are 1.834 and 1.234, respectively, while force values in elements of the diagonal cable linked to the highest node are in an ordered manner, starting from the lowest, 1,547; 1,434; 1,391; 1,394; 1,435; 1,515; 1,637; 1,834. In edge cables, the maximal and the minimal force values are 22.924 and 19.966 , respectively.

For the sake of comparison, in Figure 18.c, the minimal network from Figure 3.b was placed over the network defined by the force density method from Figure 18.a, while in Figure 18.d), the network from Figure 9.b was placed over the network form Figure 18.b. As indicated in the cited paragraph from paper [6], the form obtained by the force density method is just "a start in the formfinding process". It is usually indicated in literature that a number of attempts is needed to find force density values that provide an acceptable solution, but more detailed recommendations are lacking.

A systematic procedure by which various requirements specified in sections 4 and 5 can be met by an iterative use of the force density method will be introduced in the following section. The analogy of this method and the displacement method described in paper [24] is applied in the program implementation of the force density method.

\section{Iterative use of the force density method}

In paper [8], the authors describe "an iterative procedure devoted to the calculation of a uniform tension net and consequently a minimal length net". In the $k^{\text {th }}$-step of iteration, the force density in the element $(i, j)$ is calculated according to the expression

$q_{i, j}^{(k)}=q_{i, j}^{(k-1)} \frac{\bar{S}}{S_{i, j}^{(k-1)}}$

where
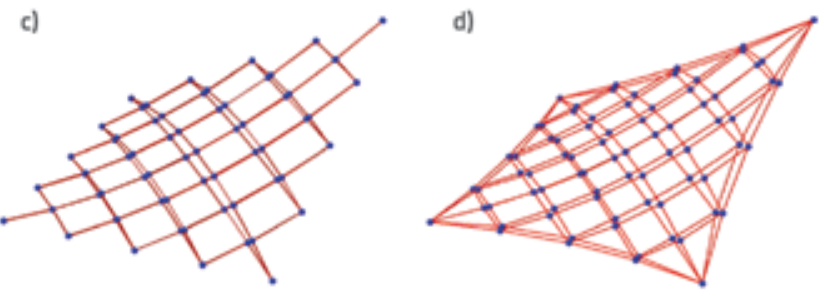

Figure 18. a) and b) Nets formed by the force density method. c) and d) Comparison with minimal networks 
$q_{i j}^{(k-1)}$ - force density in the preceding step,

$S_{i, j}^{(k-1)}$ - force value calculated in the preceding step,

$\bar{S}^{1, j}$ - required force value.

This expression can be explained in the following way:

Lets assume that the force value $S_{i,}^{(k-1)}$, calculated in the $(k-1)$ th step of the iteration, is different from the required value $\bar{S}$. The required value can be obtained by multiplying the value $S_{i, j}^{(k-1)}$ with the coefficient $S_{i, j}^{(k)}, \bar{S}=S_{i, j}^{(k)} S_{i, j}^{(k-1)}$, so that the $S_{i, j}^{(k)}$ $=\bar{S} / S_{i, j}^{(k-1)}$. Once coordinates of nodes and lengths $e_{i, j}^{(k)}$ are calculated in the k-th step, the new force value is $S_{i, j}^{(k)}=q_{i, j}^{(k)} e_{j}^{(k)}$. If the length of the element does not change, then we have $S_{i, j}^{(k)}=q_{i, j}^{(k)} e_{i j}^{(k-1)}$. Furthermore, if the $S_{i, j}^{(k)}$ is the required value, then we have $S_{i, j}^{(k)}=S_{i, j}^{(k)} S_{i, j}^{(k-1)}=S_{i, j}^{(k)} q_{i, j}^{(k-1)} e_{i, j}^{(k-1)}$. By comparison with the preceding expression we obtain $q_{i, j}^{(k)}=s_{i, j}^{(k)} q_{i, j}^{(k-1)}$. Consequently, if the length of the element does not change, the force density $q_{i, j}^{(k)}=q_{i, j}{ }^{(k-1)} \bar{S} / S_{i, j}^{(k-1)}$ immediately provides the required force value. However, the change in force density most often results in the change of equilibrium configuration of the network, and so the lengths of the elements also change. An iterative calculation is therefore needed so that the required value can gradually be approached.

The preceding explanation can be summarized as follows: according to definition given in expression (12), the force density $q_{i, j}$ is proportional to the force value $S_{i, j}$ and so, with an unchanged element length, the ratio of forces in two iteration steps is equal to the force density ratio.

In their examples, authors limit their discussion to the nets with "rigid" edges - the procedure does not enable control of force values in edge cables, nor does it provide for different force values in different cables.

Nevertheless, the described procedure can easily be extended: if we wish to achieve different force values in different elements, then the force density in the element $(i, \lambda)$ will be calculated in the k-th step of iteration according to the expression

$q_{i, j}^{(k)}=q_{i, j}^{(k-1)} \frac{\overline{S_{i, j}}}{S_{i, j}^{(k-1)}}$

At that, the $\bar{S}_{i j}$ is the required force value in that element, which can be different from force values in other elements $[9,10]$. Obviously, if we wish to form a natural generalised minimal net, then we have to assign equal force values in all elements of individual cables. As $q_{i, j} / S_{i, j}=1 / e_{i, j^{\prime}}$ the force density can also be calculated according to the following expression:

$q_{i, j}^{(k)}=\frac{\overline{S_{i, j}}}{\ell_{i, j}^{(k-1)}}$

Furthermore, the specified length $\bar{\ell}_{\mathrm{i}, \mathrm{j}}$ of the element $(i, \lambda)$ will be obtained by calculating the force density in the element according to the expression

$q_{i, j}^{(k)}=\frac{S_{i, j}^{(k-1)}}{\bar{\ell}_{i, j}}$
Namely, the force density is, according to the definition, inversely proportional to the element length and so, with the unchanged force value, we have $q_{i, j}^{(k)} / q_{i, j}^{(k-1)}=e_{i, j}^{(k-1)} / l_{i, j}^{(k)}$. That is why the coefficient by which the force density form the preceding step should be multiplied is $\bar{S}_{i j}(k-1) / \bar{\ell}_{\mathrm{i}, j^{\prime}}$ and from

$q_{i, j}^{(k)}=q_{i, j}^{(k-1)} \frac{\ell_{i, j}^{(k-1)}}{\bar{\ell}_{i, j}}$

and $S_{i, j}=q_{i, j} e_{i, j}$ we arrive to the expression (15).

The above described iterative application of the force density method presents a number of advantages. First of all, the procedure converges toward the required solution through a set of equilibrium configurations. Therefore, even if the iterative procedure is interrupted before the specified conditions have been met with sufficient accuracy, we have still obtained a net that is in equilibrium. Unlike that, in other iterative procedures, such as the Newton-Krylov procedure, only the final solution is in equilibrium. Configurations obtained in preceding steps are unbalanced approximations of the required form. The second advantage is that not only the specified forces but also the specified element lengths are obtained without introducing Lagrange multipliers. And, thirdly, as we have mentioned in the preceding section, the first approximation of the solution need not be assumed, namely because equation systems are linear, and can therefore be solved with direct procedures. In addition, the procedure is very rapid. Although it could be said that it converges slowly as, for instance, 128 and 299 steps were needed to find the form of the net shown in 3.b) and 9.b), the calculations lasted no more than $3.55 \mathrm{~s}$ and $14.40 \mathrm{~s}$, respectively. The iteration procedure interrupts when an absolute value of the maximum difference between the calculated value and required value (force in element or element length) is less than $110^{-5}$.

\section{Conclusions}

The form finding problem is formulated as a variational problem aimed at determining stationary points of specific functions, and so minimal nets and generalised minimal nets can be regarded as possible solutions. Minimal nets are equilibrium forms if the distribution of prestressing force values is uniform [8] while the generalisation, which can also be formulated as a minimisation problem, is the possibility of assigning various force values in net elements [9, 10].

Kinematic constraints that enable assignment of lengths to selected elements are introduced as an additional generalisation. The variational formulation of this generalisation, involving the use of Lagrange multipliers, is the saddle point problem. Numerical experiments have shown that the speed of convergence, and the very convergence of "traditional" iterative procedures, have been greatly disrupted. Numerical solution of a traditional problem of variational calculation, the Steiner's problem, is analyzed so as to provide a vivid explanation of behaviour of such procedures. 
Finally, the iterative use of the force density method is presented. Here, force densities are determined for a given step based on specified conditions and results from the preceding step. In addition to known force-density expressions that enable shaping of minimal nets and generalised minimal nets with various force values in elements [9, 10], additional force density expressions are introduced for meeting kinematic constraints which prevent change in length of selected elements. A series of numerical experiments has revealed that the iterative use of the force density method is an adaptable, efficient and reliable tool for finding form of flexible cable structures.

\section{REFERENCES}

[1] Nerdinger, W. (ed.): Frei Otto, Complete Works - Lightweight Construction, Natural Design, Birkhäuser, Basel, 2005

[2] Veenendaal, D.; Block, P.: An overview and comparison of structural form finding methods for general networks, International Journal of Solids and Structures, 49 (2012), pp. 3741-3753

[3] Tibert, G.: Numerical Analysis of Cable Roof Structures, Royal Institute of Technology, Stockholm, 1999

[4] Argyris, J. H.; Angelopoulos, T.; Bichat, B.: A general method for the shape finding of lightweight tension structures, Computer Methods in Applied Mechanics and Engineering, 3 (1974), pp. 135-149

[5] Schek, H.-J.: The force density method for form finding and computation of computation of general networks, Computer Methods in Applied Mechanics and Engineering, 3 (1974), pp. 115-134

[6] Linkwitz, K.: About formfinding of double-curved structures, Engineering Structures, 21 (1999), pp. 709-718

[7] Linkwitz, K.; Bahndorf, J.; Hördt, R.; Singer, P.; Ströbel, D.: Least squares methods in non-linear formfinding and analysis of prestressed and hanging nets, Design and Realisation of Textile Roofs (workshop), Technische Universität, Institut für Geodäsie und Photogrammetrie, Berlin, June 15-17, 1995, pp. C/1-C/11

[8] Maurin, B.; Motro, R.: Investigation of minimal forms with conjugate gradient method, International Journal of Solids and Structures, 38 (2001), pp. 2387-2399

[9] Dvornik, J.; Lazarević, D.: Prednapregnute gipke konstrukcije od užadi i tkanine, Građevinar, 47 (1995), str. 185-199

[10] Dvornik, J.; Lazarević, D.: Viseće konstrukcije od platna i užadi, Građevni godišnjak '97, HDGl, Zagreb, 1997., str. 239-297

[11] Haber, R. B.; Abel, J. F.: Initial equilibrium solution methods for cable reinforced membranes. Part I - Formulations, Computer Methods in Applied Mechanics and Engineering, 30 (1982), pp. 263-284

[12] SciPy. Scientific Tools for Python (Version 0.12), The Scipy Community, 2013, http://scipy.org

[13] Stein, W. A. et al.: Sage Mathematics Software (Version 5.9), The Sage Development Team, 2013, http:/ /www.sagemath.org
[14] Eröcal, B.; Stein, W.: The Sage Project: Unifying Free Mathematical Software to Create a Viable Alternative to Magma, Maple, Mathematica and Matlab, ICMS 2010: Proceedings of the Third International Congress on Mathematical Software, (eds. Fukuda, K.; van der Hoeven, J.; Joswig, M.; Takayama, N.), Springer, 2010., pp. 12-27

[15] Kelley, C. T.: Iterative Methods for Linear and Nonlinear Equations, Society for Industrial and Applied Mathematics, Philadelphia, 1995

[16] Knoll, D. A.; Keyes, D. E.: Jacobian-free Newton-Krylov methods: a survey of approaches and applications, Journal of Computational Physics, 193 (2004), pp. 357-397

[17] Bićanić, N.; Johnson, K. H.: Who was '-Raphson'? International Journal for Numerical Methods in Engineering, 14 (1979), pp. 148-152

[18] Baker, A. H.; Jessup, E. R.; Manteuffel, T.: A Technique for Accelerating the Convergence of Restarted GMRES, SIAM Journal on Matrix Analysis and Applications, 26 (2005), pp. 962--984

[19] Buchholdt, H. A.: An Introduction to Cable Roof Structures, Cambridge University Press, Cambridge, 1985

[20] Lanczos, C.: The Variational Principles of Mechanics, University of Toronto Press, Toronto, 1970 (republication: Dover, New York, 1986)

[21] Hildebrandt, S.; Tromba, A.: The Parsimonious Universe. Shape and Form in the Natural World, Springer, New York, 1996

[22] Dvornik, J.; Lazarević, D.; Fresl, K.: The Fractal Nature of the Form Finding Equations, Computational mechanics in the UK 11th Annual Conference of the Association for Computational Mechanics in Engineering (United Kingdom) (ed. Wheel, M. A.), University of Strathclyde, Glasgow, 2003, pp. 201-204

[23] Dvornik, J.; Lazarević, D.: Fractals and formfinding - magic with real numbers, Engineering Modelling, 16 (2003), pp. 1-11

[24] Gidak, P.; FresI, K.: Programming the force density method, IASSAPCS 2012, From spatial structures to space structures, (ed. Seung Deog, K.), Seoul, South Korea, 2012, p. 197 (abstract, full paper on CD) 